\title{
Autonomous Vehicles as Local Traffic Optimizers
}

\author{
Ashna Bhatia ${ }^{1}$, Jordan Ivanchev ${ }^{1,2(\bowtie)}$, David Eckhoff ${ }^{1,2}$, and Alois Knoll ${ }^{2,3}$ \\ 1 TUMCREATE, 1 Create Way, Singapore 138602, Singapore \\ \{ashna.bhatia, jordan.ivanchev, david.eckhoff\}@tum-create.edu.sg \\ 2 Technical University of Munich, 3 Boltzmannstr., 85747 Munich, Germany \\ knoll@in.tum.de \\ 3 Nanyang Technological University, 50 Nanyang Avenue, \\ Singapore 639798, Singapore
}

\begin{abstract}
This paper explores the interaction between autonomous and human-driven cars on a microscopic level using an agent-based traffic simulator. More specifically, it deals with the design of driving logic models of "socially-aware" autonomous vehicles that can improve the performance of surrounding vehicles on the road. Congestion waves, which are created as a result of an abrupt stopping or a car joining a highway, are a known phenomenon in current traffic systems. Experiments performed, demonstrate how the presence of intelligent social vehicles on the road can reduce such effects by acting as a flexible medium between humandriven cars. Metrics to evaluate benefits ot our AV behaviour models under various states of traffic conditions/congestion are also proposed. Finally, results showing the effectiveness of these models are presented.
\end{abstract}

Keywords: Autonomous vehicles $\cdot$ Mixed traffic agent-based simulation · Driver models

\section{Introduction}

The current trends of Intelligent Transport Systems (ITS) and their applications concerning intelligent cars include advancements in Adaptive Cruise Control (ACC), Obstacle Warning, Avoidance Mechanism, Lane Detection and Collision Notification, which all contribute towards making travel more comfortable and safe [1]. In [2,3] it is reiterated that sustainable ITS application will enable the reduction in carbon dioxide and heat emission as traffic volume is reduced and managed. Research and development in the sector of Autonomous Vehicles $(\mathrm{AVs})$ is being done worldwide. Benefits from them, such as more independent mobility for affluent non-drivers, may begin sooner rather than later. However, most impacts concerning improvements in safety, sustainability and comfort will only be significant when AVs become common and affordable, which might take another 20 to 30 years [4].

Consequential to these technologies, expectations and predicted trends, copious evaluations have been made on the impact of penetrating AVs into the road

(C) Springer Nature Switzerland AG 2020

V. V. Krzhizhanovskaya et al. (Eds.): ICCS 2020, LNCS 12137, pp. 499-512, 2020.

https://doi.org/10.1007/978-3-030-50371-0_37 
transport sector. The research done in [5] shows how vehicle-to-vehicle communication, can make a difference in factors such as fuel consumption, driver safety and convenience. A mixed simulation scenario with 3 types of car models; manual, ACC, and Cooperative Adaptive Cruise Control (CACC) is analysed in [6]. The experiments carried out, analyse optimal platoon sizes feasible due to the presence of CACC, that enhance the traffic flow.

Promising improvements using V2I communications can be seen in the work done on Variable Speed Limits in [7] wherein a control algorithm is presented, which simultaneously maximizes the mobility, safety and environmental impact by finding a balanced trade-off. Another multi-objective approach considering efficiency, comfort, throughput, and safety is presented in [8] where a simplistic mixed traffic scenario is considered and optimal parametrizations of known carfollowing models are found.

An alternative approach to maximizing the benefits of autonomous mobility is to separate the AVs from the rest of the traffic. A macroscopic evaluation of a dedicated AV lane policy on all highways for the city of Singapore has been presented in [9]. Additionally, a microscopic analysis of replacing High Occupancy Vehicle lanes with dedicated AV lanes has been performed in [10]. Those studies, however, model expected AV behaviour rather than trying to design its logic so that it benefits traffic conditions, which is the focus of our work.

It is clear that, mixed traffic scenarios will be unavoidable with the onset of AV acceptance. Various questions arise in this scenario; 1) how should an AV behave in order to optimize the impact of its presence? 2) To what extent will the behaviour impact surrounding human-driven vehicles? 3) What traffic parameters can it improve (safety, comfort, throughput)? 4) How safe is the interaction between human controlled and automated vehicles? 5) Will additional information regarding surrounding vehicles in an autonomous vehicles' neighbourhood, help to improve traffic conditions (as opposed to only considering the vehicles in front and behind)?

In order to begin addressing those questions in an efficient and safe manner, we suggest to use a modelling and simulation approach. The main challenge when it comes to simulating mixed traffic is capturing the interactions between vehicles. This requires the usage of an agent-based simulation approach where behavioural models are responsible for the decisions that agents take on the road, given the environment around them. In our work we use a multi-agent traffic simulation environment for designing and testing the control logic of AV driver models that aim to improve traffic conditions for all traffic participants in terms of safety, fuel consumption and throughput. This allows us to fully determine and control the traffic environment, and to run all different scenarios we need, in order to find beneficial autonomous vehicle control parameters.

The contributions of this paper can be summarized as follows:

- Design of socially aware autonomous vehicle behaviour models

- Design of metrics to evaluate the performance of these models in terms of efficiency, safety, and traffic robustness 
- Analysis of agent population subgroup behaviour and performance to understand the mechanism behind the improvement of traffic conditions.

\section{Simulation Framework}

Since mixed traffic conditions do not currently exist in the real world, using simulation seems as a viable approach to study mixed traffic. For the purposes of this research we have chosen the CityMoS simulation framework [11], which is microscopic and agent-based in nature. The behaviour of these agents (vehicles) is programmed using driver models which are introduced in the subsequent subsection. More specifically, we use the BEHAVE (Behaviour Evaluation of Human-driven and Autonomous VEhicles) tool [12], powered by the discrete-time based CityMoS engine, which is designed specifically for studying the interactions between $\mathrm{AVs}$ and human-driven vehicles.

Driver Models. Various car-following and lane-changing driver models have already been proposed in order to mimic the behaviour and dynamics of vehicles on the road in a simulation environment. Few of the well-known ones are the Gipps' Model [13], the Intelligent Driver Model(IDM) [14], the MOBIL Lane Changing Model [15], etc.

Driver models like IDM take as input the information about the vehicle in front and compute as output the forward acceleration, which will be applied. The models are parametrized by the personal preferences of the driver such as: timeheadway, acceleration and deceleration components, minimum safety distance gaps etc. These parameters can be calibrated in order to closely match a given real-world data set.

The aspects of driving that make AVs different from humans that have been assumed in this work include almost no reaction time and perfect perception which actually is a fair description of the IDM. For this reason, we use it to model naive non-social AVs, and benchmark their performance against more elaborate models that we design later in the paper. In order to study the vehicle interactions in mixed-traffic conditions (human-driven and autonomous vehicles), an adequate model that represents human-driven vehicles should be identified as well. We use the Human Driver Model (HDM) [16], which is an extension of the work carried out for the IDM.

The IDM stands in the core of all model design in this work since it: 1 ) is the benchmarking model we use for non-social AVs, 2) is the model which is extended to yield the human driver model (HDM), and 3) is the model we extend to build our social autonomous vehicle models. It is a well established car-following model that is simplistic and realistic in terms of formulation and simulation, respectively. The acceleration (Eq. 1) of a vehicle $\alpha$ at a given time $t$ is calculated using the continuous acceleration function $\dot{v}_{\alpha}\left(s_{\alpha}, v_{\alpha}, \Delta v_{\alpha}\right)$ depending on the vehicle's actual velocity $v_{\alpha}(t)$, the net distance $s_{\alpha}$ and the approaching speed $\Delta v_{\alpha}(t)$. 


$$
\dot{v}_{\alpha}=a\left[1-\left(\frac{v_{\alpha}}{v_{0}}\right)^{\delta}-\left(\frac{s^{*}\left(v_{\alpha}, \Delta v_{\alpha}\right)}{s_{\alpha}}\right)^{2}\right]
$$

with

$$
s^{*}\left(v_{\alpha}, \Delta v_{\alpha}\right)=s_{0}+v_{\alpha} T+\frac{v_{\alpha} \Delta v_{\alpha}}{2 \sqrt{a b}}
$$

where $s_{0}$ corresponds to the desired minimum distance gap to the preceding vehicle, $T$ to the time gap to safely come to a halt without crashing into the vehicle in front, and $b$ to the vehicle's comfortable deceleration. Here, $v_{0}$ corresponds to the vehicles desired velocity, $a$ corresponds to the vehicles maximum acceleration, and $\delta$ determines how the acceleration decreases once the desired velocity i.e $v_{0}$, is reached.

To incorporate more realistic behaviour into the IDM, it was extended to derive the Human Driver Model (HDM) in [16] . Human-like behaviour is integrated by taking into consideration the following destabilizing and stabilizing factors.

1. Finite reaction time: Human drivers have a certain finite reaction time $T^{\prime}$ to events occurring on the road. By computing the acceleration at time $t-T^{\prime}$ instead of at time $t$, an effect equivalent to delayed response to stimuli on the road can be achieved.

2. Estimation errors: As shown in Eq. 1 acceleration is a function of the distance to the preceding vehicle $s$ and the approach speed $\Delta v$. Those inputs to the model typically are the perfect measurements provided by the simulator, however, as a human driver is certainly plausible that some perception errors will be introduced. The HDM models this, using stochastic noise introduced by a Weiner process that leads to time-correlated fluctuations of the acceleration.

3. Temporal anticipation: Human drivers can be intuitive enough to anticipate their reaction time and the evolution of traffic within small time windows. To represent this awareness, of future net distance and future velocity, HDM uses constant acceleration and velocity projections to determine their current values, respectively.

4. Spatial anticipation: The receptivity range of the human driver can be extended to more than one preceding vehicle on accord of spatial perceptiveness. The IDM considers relative measures to only one vehicle ahead, as can be seen in its deceleration determining component. The HDM expands the interaction term by taking into account multiple nearest preceding vehicles.

The first two factors are responsible for destabilizing the traffic conditions and the last two being anticipatory in nature, contribute towards the stability. In our studies we use parameter values specified by the authors of the HDM for all additional parameters introduced in the model which achieve a balance between the 4 additional aspects of the model.

\section{Social AV Models}

One of the reasons IDM is widely used is because it has succeeded in showing robustness and aptness by qualitatively reproducing results from empirical 
data in simulation environments. This was achieved through varying mostly one parameter that the model is quite sensitive to, namely, the time-headway [14], or the distance, in terms of time, that an agent considers to be safe. Hence, in our models, which are based on IDM, we modulate this parameter as well to steer the behaviour of our agents. Consequently, the goal is to formulate models that control AVs and improve on the following measures:

1. Performance: in terms of total traffic capacity and average fuel consumption

2. Safety: in terms of accidents caused in the traffic scenario.

3. Resilience: in terms of response and recovery from perturbations that cause congestion waves.

Modelling the First Social AV Model - SAV. By exploring the interaction between mixed (IDM and HDM) agent populations using BEHAVE, the effect of the time-headway parameter was analysed in details. It was observed that as the time-headway value was increased, the overall traffic conditions became more stable and safe. This comes at the cost of reduced throughput and overall average velocity.

The central idea behind the first design of a social AV model is to provide information about more vehicles in its vicinity. Adhering to the single-lane carfollowing set-up, the idea is to incorporate in an AVs decision making logic, information about not just the preceding vehicle (as the IDM does), but also of the following one. The hypothesis being; an AV that is aware of the distance to its following and preceding vehicles, can make decisions regarding its own dynamics which can benefit the entire traffic situation.

The intended behaviour of this AV is designed such that, the AV adjusts its own time-headway parameter value so that it is equidistant from the vehicle ahead and the vehicle behind. The maximum and minimum permissible value of the time-headway is set to be $2.5 \mathrm{~s}$ and $0.5 \mathrm{~s}$, respectively.

The AV is spatially aware and knows the position of the vehicle in front $\left(x_{i-1}\right)$ and the vehicle behind $\left(x_{i+1}\right)$, along with its own position $\left(x_{i}\right)$. The modulation factor $\mu$ as shown in Eq. 3 is the difference of the current position of the vehicle from the midpoint of the leading and following vehicles. The vehicle in front has a greater position than the vehicle behind. These positions are relative to the coordinates of a highway beginning at position 0 .

$$
\mu=x_{i}-\left(\frac{x_{i-1}+x_{i+1}}{2}\right)
$$

If the AV is closer to the vehicle ahead, $\mu$ will be positive and vice versa. Taking the logistic sigmoid of this metric, (Eq. 4) and bounding it in the range of the set limit values for the time-headway, results in a greater value for $T$ as the AV gets closer to the vehicle ahead. This means it will now aim at increasing the distance ahead by increasing its time-headway proportionally, as per the following equation.

$$
T=\left(T_{\max }-T_{\min }\right) * S(\mu)+T_{\min }
$$


With this underlying model logic, the AV continuously modulates its own timeheadway based on its difference from the midpoint and the general equation of the IDM (Eq. 1). It always aims to be in the centre of its remote neighbourhood to reduce the overall deviation in spacing between vehicles. From this point forward, the model obeying this design will be referred to as the SAV i.e. Social Autonomous Vehicle.

Extending the SAV - SAVE. Given the advancements in V2V and V2I communications, it is plausible that an AV is capable of having more information regarding its neighbourhood, than what is provided to the SAV. Thus, in our second approach, the Social Autonomous Vehicle- Extended (SAVE) model, incorporates additional information regarding the traffic dynamics by expanding the neighbourhood awareness of the AV with a congestion predictive control parameter.

Similar to the logic of the SAV model, the SAVE model aims to achieve improvement of throughput, safety and traffic resilience by modulating the timeheadway parameter. Instead of relying solely on the relative positions, this AV model was intended to understand the traffic dynamics better, for which it uses a congestion predictive control parameter called time-to-next (TTN). These values facilitate a prediction into the remote future by stating the time it will take for a vehicle to reach or catch-up with the next vehicle, given its current speed and the current distance to the vehicle ahead, as shown in this equation:

$$
T T N=\frac{s_{\alpha \beta}}{v_{\alpha}}
$$

The AV is provided with two proximity-based sorted lists containing this $T T N$ information, for the vehicles ahead and behind, respectively. The length $N$ of each list denotes the size of the neighbourhood under consideration.

Before responding intelligently, an AV needs to evaluate which traffic condition it is currently in: entering a congestion wave, or exiting it. One way to do that is by using weighted means of $T T N$ values calculated from the two sorted lists. They give a look-ahead and look-back in traffic distribution, while giving greater priority to closer vehicles.

$W_{1}$ represents the relative weighted proximity (in terms of $T T N$ values) to the clusters. It is set according to the difference between two exponentially weighted means(one concerning the cluster of vehicles ahead, and the other concerning the cluster behind), as can be seen in Eq. 6. A positive value of $W_{1}$ means that the cluster ahead,as a whole is closer to the vehicle in question, as compared to the cluster of vehicles behind it.

$$
W_{1}=\sum_{i=1}^{N} \frac{2^{N-i}}{\sum_{i=1}^{N} 2^{N-i}}\left(T T N^{\text {behind }}-T T N^{\text {ahead }}\right)
$$

$W_{2}$ is represents compactness of clusters using a relative congestion factor. First, we compute the deviation of the vehicles from the weighted mean of the 
cluster they belong to. Second, we subtract this value of the cluster ahead, from the cluster behind (Eq.7). A low deviation from weighted mean implies that the vehicles in the cluster are similar in $T T N$ values to the first vehicle in the cluster. It is to be noted that the first vehicle considered here, is the one closest to the SAVE in question. Hence, if the deviation in weighted means in the cluster ahead is lower than that of the cluster behind, $W_{2}$ is positive and the cars ahead are more closely packed compared to the ones behind.

$$
W_{2}=D\left(T T N_{\text {behind }}\right)-D\left(T T N_{\text {ahead }}\right)
$$

where,

$$
D(T T N)=\sqrt{\frac{\sum_{i=1}^{N}\left(\bar{x}_{w}-T T N_{i}\right)^{2}}{N}}
$$

$W_{3}$ is used to make the SAVE more sensitive to its immediate neighbourhood. It uses the $T T N$ values of the vehicle immediately ahead, and immediately behind. It tries to help the SAVE model achieve an average of these TT $N$ values. If $W_{3}$ is positive, the current $T T N$ value is smaller than average, and closer in proximity to the vehicle ahead. Thus the SAVE uses this weight to equalize spacing in its close proximity region. This means, when the weight is positive the current SAVE will increase its time-headway, and thus try falling back in the relative proximity centre.

$$
W_{3}=\left(\frac{T T N_{i-1}+T T N_{i+1}}{2}\right)-T T N_{i}
$$

All three weights when positive intend to increase the time-headway, and vice-versa when negative. Experiments show that a non-linear combination of the three weights, in this case multiplying them (Eq. 10), results in better modulation of time-headway than a simple addition.

$$
\mu=W_{1} * W_{2} * W_{3}
$$

- Increase in time-headway: By increasing the time-headway of a vehicle we increases its distance from the vehicle ahead. This is ideal when we all the weights are positive. When the cluster ahead is more compact.

\section{Experiments}

In our experiments, we assume that half of the agent population is human driven and modelled by HDM. The other half consists of AVs that we model with either IDM, SAV or SAVE. The BEHAVE simulator was used to simulate a $50 \mathrm{~km}$ single-lane highway with vehicle density of 0.067 vehicles per meter. Each simulation run lasted $45 \mathrm{~min}$ in real-time, which is significantly faster in the multi-core enabled simulator having high speed processing capabilities. Results derived from the experiments were averaged over 20 simulation runs, each. 
Experiment 1 - Congested Traffic Flow. The aim of this experiment is to analyse the interactions of AVs with HDMs in slow moving traffic and test the hypothesis that their presence can improve overall traffic conditions. This experiment is performed on 4 group settings. The first 3 are under mixed traffic conditions with $50 \% \mathrm{HDMs}$ and $50 \%$ of each of the $3 \mathrm{AVs}$. The last group represents the evaluation of only HDMs by themselves, i.e without the presence of any AVs.

The performance of the models is evaluated in terms of the achieved throughput, indicative of the capacity of the road, and the average absolute acceleration, indicative of the smoothness of traffic flow and the fuel consumption.

1. Throughput: The throughput of our traffic system is collected as the average number of cars passing through a chosen set point on the highway in a time period of $10 \mathrm{~min}$.

2. Average Absolute Acceleration: The average fuel consumption of a vehicle, increases with increase in levels of acceleration among vehicles. The more the vehicles on the road accelerate the more fuel they will use. The stability of traffic and consequently ride comfort is also related to the acceleration profile, however, also includes the amount of deceleration that is performed. In order to capture both those aspects the average of the absolute value of the vehicles' accelerations is used as a metric. The absolute value of acceleration of each vehicle in a simulation run is summed up and averaged at every time-stamp.

Results from Fig. 1 show that the SAV models achieved the highest throughput. The scenario of a pure HDM population produces less than half the throughput.

The results from Fig. 2, indicate that the setting comprising only HDMs results in oscillatory traffic conditions that exude larger amount of fuel on average per vehicle while the SAVE vehicles provide the least oscillatory, and thus efficient, traffic conditions.

Experiment 2 - Perturbed Congested Traffic. The aim of this experiment is to analyse the traffic conditions under the presence of stop-and-go congestion waves. The extent to which these waves can affect the vehicles in terms of safety, and the traffic dynamics in terms of stability.

Similar to the previous experiment, 4 group settings were used i.e 3 with mixed dense traffic conditions and the last one representing only human drivers. Each simulation run had a duration of $45 \mathrm{~min}$. After one-third of the run was done, a trigger event was invoked that caused the leading few vehicles to stop completely for the average duration of a traffic signal (1 min in real-time), post which they began to accelerate as per their own preferred velocity and, as permissible by the road density and traffic conditions. This trigger causes the phenomena of stop-and-go congestion waves which are observed to propagate downstream (away from the source of perturbation).

A congested traffic with perturbations gives rise to the following important considerations: 


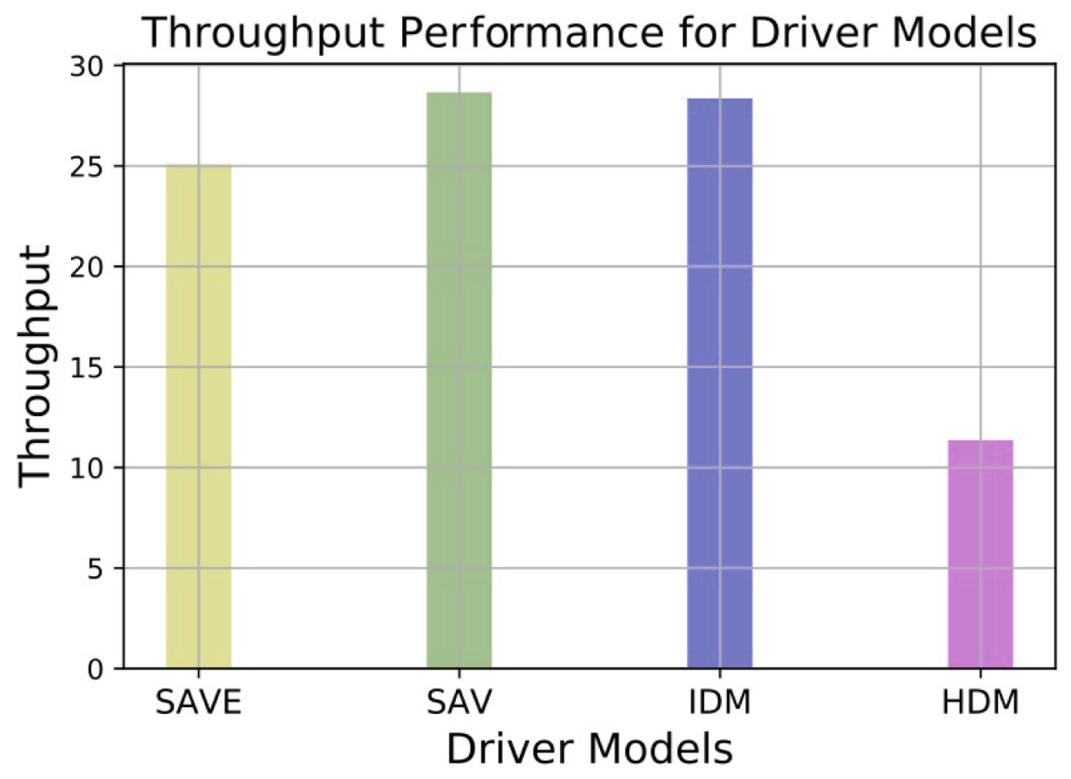

Fig. 1. Throughput

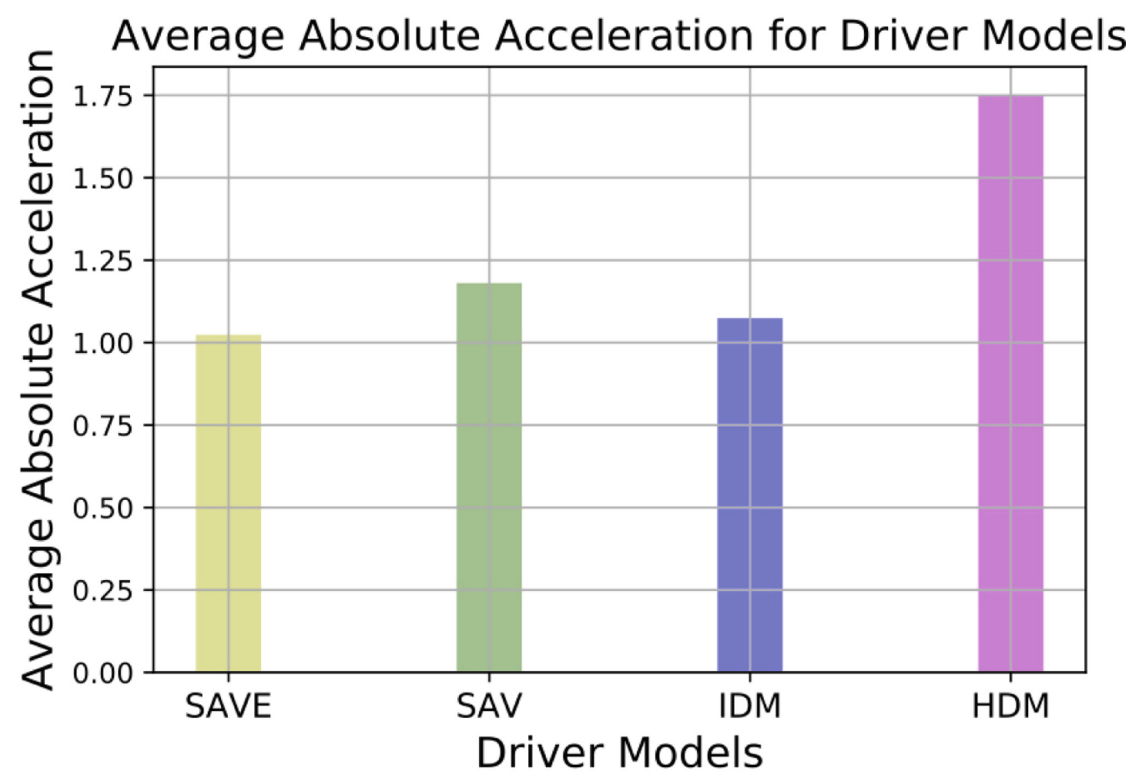

Fig. 2. Average absolute acceleration 
1. Safety: While the AV driver models i.e the IDM, SAV and SAVE are crashfree, the human driver model HDM is not. The perturbation in traffic helps to magnify the safety issues in mixed traffic.

2. Resilience: The ability of a system to revert back to its state of equilibrium after being perturbed, shows its resilience to change. A system with greater resilience can be characterized as more robust or stable. The aim of this comparative experiment is to realize which of the $3 \mathrm{AV}$ models could result in a more stable traffic dynamics, and how long would it take to regain their equilibrium post perturbation.

The number of crashes for 100 vehicles per $1.5 \mathrm{~km}$ in $45 \mathrm{~min}$ of slow moving traffic, is recorded for each of the model set-ups. As can be seen from Fig. 3, the number of crashes significantly reduced (by $70 \%$ to $80 \%$ ) when AVs were mixed in. On an average of 20 simulations, the set-up with AVs controlled by the SAVE model showed to be the safest scenarios. A system exhibiting stable traffic flow, can be described pertaining to vehicles with minimal change in values of acceleration, and also equal spacing from one-an-other, making maximum use of road capacity. For the sake of representing these two criteria, macroscopic parameters $A$ and $D$, are used. The Average Absolute Acceleration $A$ represents the non-smoothness of traffic conditions. The deviation in average spacing of the vehicles on the road $D$ gives an idea of about the degree of heterogeneity in the distances between the vehicles. This is an indication of the magnitude of the stop-and-go wave created by the disturbance.

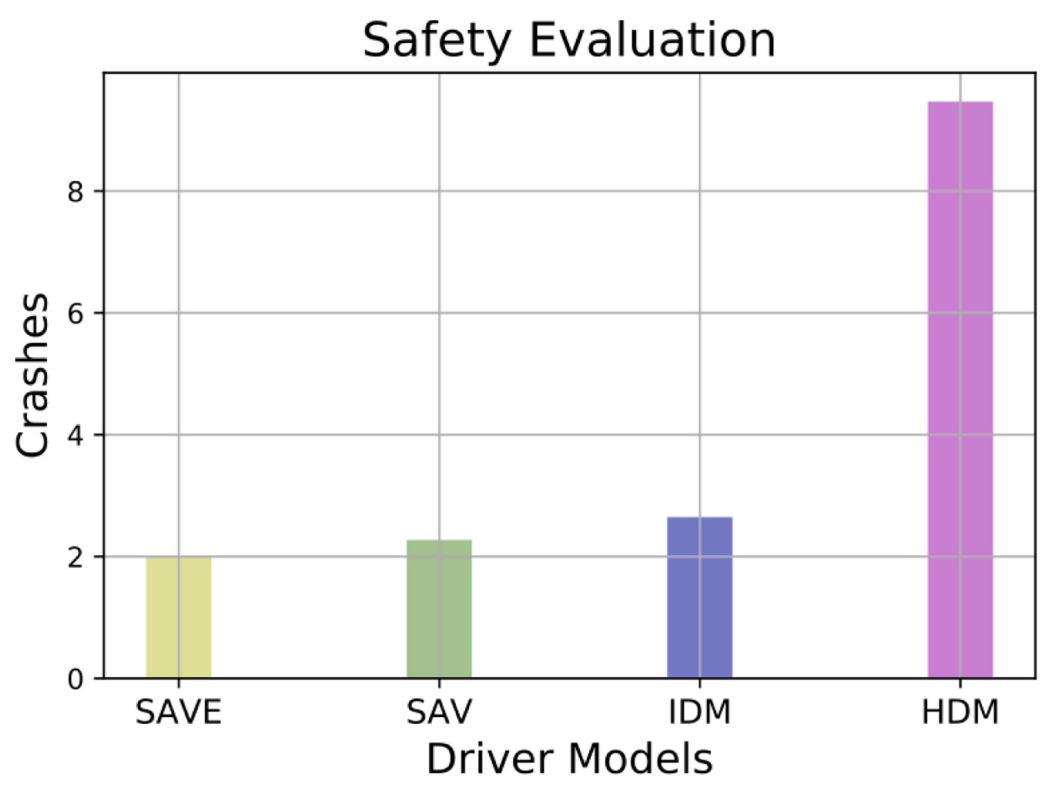

Fig. 3. Crashes $k m / \min$ 
The instability values plotted on the $y$ - axis of Fig. 4 are the product of $A$ and $D$. The higher this value, the more unstable is the system. The $x-$ axis represents the time post perturbation in minutes. Each coloured polygon shows how the system, comprising $50 \%$ of the respective AV models, reacts to the perturbation and recovers from it. The magenta coloured polygon with the greater area, corresponds to the simulation runs with HDMs only. The black dots at time 0 , are called the equilibrium offset that represent the initial equilibrium value for each model set-up. As can be seen, the equilibrium offset for SAVE models are the best and most stable. The perturbation trigger is also applied at this instant causing the peak instabilities.

As the time progresses, the system is expected to converge back to its equilibrium offset. The area under the instability vs. time curve shows how efficiently the system mitigates the effect of congestion waves. The lesser the area, the better is the recovery. To determine which model is most stable, we find the product of the equilibrium offset and the area under the curve, and term it as the instability index. The HDMs alone, as can be seen, don't seem to converge back or close to their own system equilibrium. The penetration by $50 \% \mathrm{AVs}$ has much of an alleviating effect. The instability index of SAVEs is the best, followed by SAV .

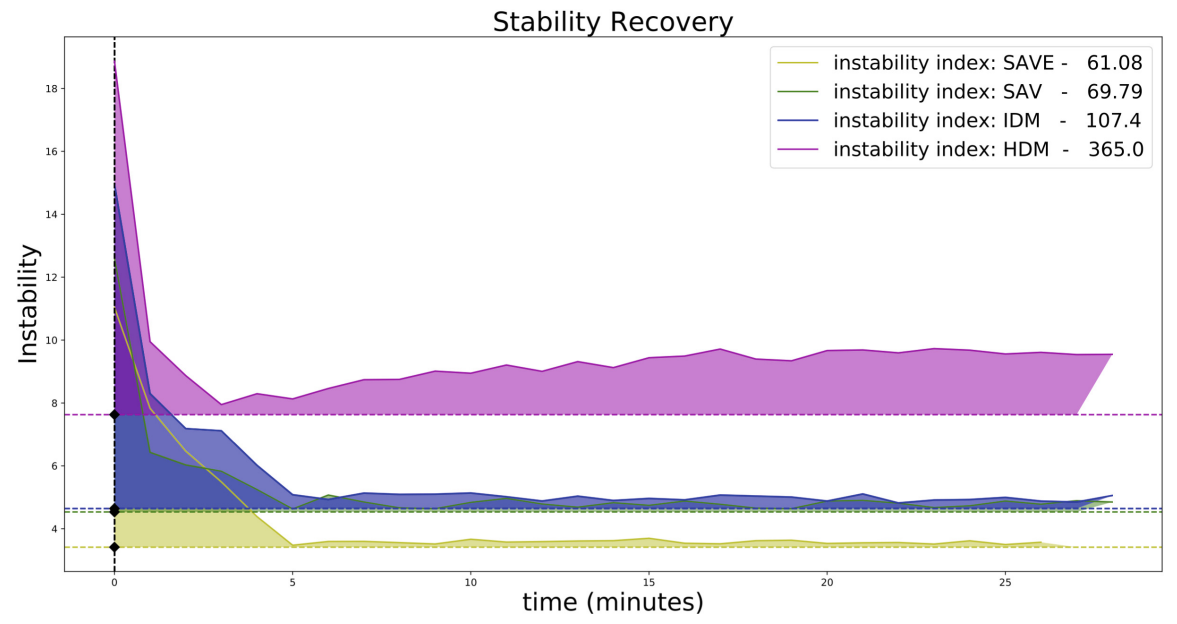

Fig. 4. Stability recovery of driver models with HDMs

The already presented evaluations and comparisons measure the overall macroscopic properties of mixed-traffic scenarios. In order to see how different vehicle populations perform in these runs, we extend the analysis done in Experiment 2 by examining the performance metrics of the two driver categories (i.e. human and autonomous) in every scenario separately. This evaluation is important as it sheds light on how the presence of AVs in a traffic scenario, could help 
bring about improvements in the performance of human drivers. The comparative approach also demonstrates which AV model can be more advantageous for this purpose and to what extent does it sacrifice its own gaining, in order to benefit the other population.

Figure 5, shows the values of the overall macroscopic parameter called Average Absolute Acceleration $A$ that is computed separately for the HDM and the $\mathrm{AV}$ models and normalized to produce a metric that we call Disturbance. By comparing the difference between these two values in every group, we observe that: 1) The overall Disturbance for the SAVE + HDM scenario results in the best collective evaluation (as shown by the black dots), and 2) The SAVs are more "altruistic" as the difference between them and the HDMs is smaller. In other words, it seems that the SAV perform active acceleration and deceleration manoeuvres in order to minimize the oscillation of the HDMs. This means that, the human drivers in the presence of SAVs will have a smoother driving experience and more efficient fuel consumption, than with SAVEs or IDMs.

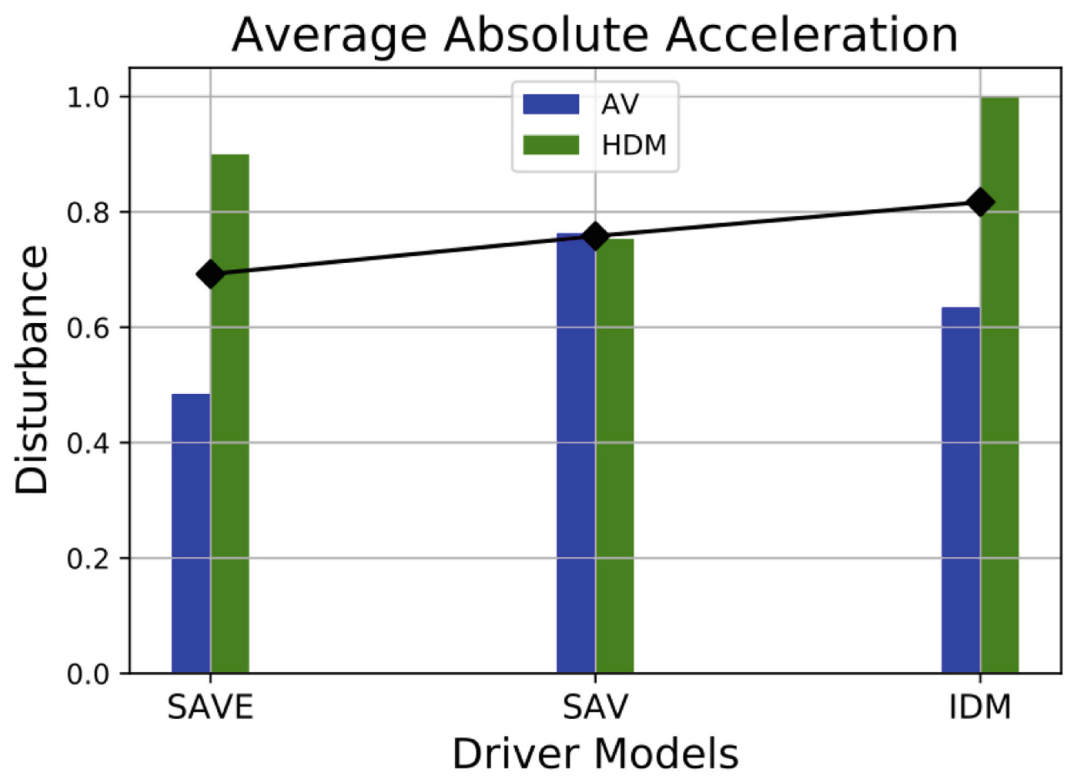

Fig. 5. Rate of deviation from equilibrium value of average absolute acceleration for experiments with 50\% HDM and 50\% AV driver models

\section{Conclusions and Future Work}

This paper analyses the interaction between $\mathrm{AVs}$ and human-driven vehicles in traffic states involving congestion and stop-and-go waves. We design AV driver models that aim at increasing the beneficial effect AVs can have on overall traffic 
properties as throughput, safety, and resilience of traffic flow against perturbations. We compare the performance of the two models that we have designed to a naive implementation of an AV that does not try to improve traffic conditions around it, modelled as an IDM, and to a scenario with only human-drivers.

Having AVs in the system improves all discussed traffic parameters. Furthermore, the SAV and SAVE models lead to improvements compared to the IDM model. The main idea behind the SAV model is to stay equidistant to the vehicles in front and behind it. This allows for quick stabilization to normal flow conditions after perturbations are introduced to the system and thus the SAV has the highest resilience score of all tested models. The SAVE model, tries to improve on traffic throughput by utilizing information about groups of vehicles in front and behind to determine whether it is approaching a local congestion zone. It achieves a better throughput than the other examined models without compromising the level of safety on the road in terms of number of accidents.

As research on AV technology advances, mixed traffic conditions will inevitably occur sooner rather than later. There are numerous concerns regarding the safe interaction between AVs and human drivers, mostly due to the unpredictability of humans. It is therefore, important to study those interactions and to design solutions which mitigate the safety risks while not compromising traffic performance. Our results demonstrate that by utilizing more information about a vehicle's surroundings, high-level control models can be designed in order to improve both safety and efficiency of the studied traffic system. This work can be considered as a first step to utilizing AVs as local traffic optimizers rather than just means of transportation.

After we have shown that dedicated strategies might improve the studied traffic characteristics, we see plenty of opportunities to extend this line of research. Reinforcement learning approaches can be used to come up with a continuously learning and adapting driver behaviour model. Model predictive control can be used to guarantee safety and continuously optimize chosen traffic characteristics. Apart from extending the variety of tools for optimizing control of car following models, we can extend those tools into lane changing decision making and overall vehicle coordination with safety and efficiency in mind. We would further like to explore a wider variety of scenarios including on and off ramps of highways, traffic junctions etc. thus also covering urban intelligent transport systems.

Acknowledgement. This work was financially supported by the Singapore National Research Foundation under its Campus for Research Excellence And Technological Enterprise (CREATE) program.

\section{References}

1. Nkoro, A., Vershinin, Y.A.: Current and future trends in applications of intelligent transport systems on cars and infrastructure. In: 2014 IEEE 17th International Conference on Intelligent Transportation Systems (ITSC), IEEE, pp. 514$519(2014)$ 
2. McDonald, M., et al.: Intelligent Transport Systems in Europe: Opportunities for Future Research. World Scientific, Singapore (2006)

3. Ivanchev, J., Fonseca, J.A.: Anthropogenic heat due to road transport: a mesoscopic assessment and mitigation potential of electric vehicles and autonomous vehicles in Singapore. Technical Report, ETH Zurich (2020)

4. Litman, T.: Autonomous Vehicle Implementation Predictions. Victoria Transport Policy Institute Victoria, Canada (2017)

5. Bergenhem, C., Hedin, E., Skarin, D., et al.: Vehicle-to-vehicle communication for a platooning system. Procedia-Soc. Behav. Sci. 48(2012), 1222-1233 (2012)

6. Zhao, L., Sun, J.: Simulation framework for vehicle platooning and car-following behaviors under connected-vehicle environment. Procedia-Soc. Behav. Sci. 96, 914924 (2013)

7. Khondaker, B., Kattan, L.: Variable speed limit: a microscopic analysis in a connected vehicle environment. Transport. Res. Part C Emerg. Technol. 58, 146-159 (2015)

8. Ivanchev, J., Eckhoff, D., Knoll, A.: System-level optimization of longitudinal acceleration of autonomous vehicles in mixed traffic. In: IEEE Intelligent Transportation Systems Conference (ITSC). IEEE 2019, pp. 1968-1974 (2019)

9. Ivanchev, J., Knoll, A., Zehe, D., Nair, S., Eckhoff, D.: A macroscopic study on dedicated highway lanes for autonomous vehicles. In: Rodrigues, J.M.F., et al. (eds.) ICCS 2019. LNCS, vol. 11536, pp. 520-533. Springer, Cham (2019). https:// doi.org/10.1007/978-3-030-22734-0_38

10. Xiao, L., Wang, M., van Arem, B.: Traffic flow impacts of converting an HOV lane into a dedicated CACC lane on a freeway corridor. IEEE Intelligent Transportation Systems Magazine (2020)

11. Zehe, D., Nair, S., Knoll, A., Eckhoff, D.: Towards citymos: a coupled city-scale mobility simulation framework. In: 5th GI/ITG KuVS Fachgespräch Inter-Vehicle Communication, p. 26 (2017)

12. Ivanchev, J., Braud, T., Eckhoff, D., Zehe, D., Knoll, A., Sangiovanni-Vincentelli, A.: On the need for novel tools and models for mixed traffic analysis. In: 26th ITS World Congress, Singapore, October 2019, to appear

13. Gipps, P.G.: A behavioural car-following model for computer simulation. Transport. Res. Part B Methodol. 15(2), 105-111 (1981)

14. Treiber, M., Hennecke, A., Helbing, D.: Congested traffic states in empirical observations and microscopic simulations. Phys. Rev. E 62(2), 1805 (2000)

15. Kesting, A., Treiber, M., Helbing, D.: General lane-changing model mobil for carfollowing models. Transport. Res. Record 1999(1), 86-94 (2007)

16. Treiber, M., Kesting, A., Helbing, D.: Delays, inaccuracies and anticipation in microscopic traffic models. Phys. A Stat. Mech. Appl. 360(1), 71-88 (2006) 